\title{
Revisionseingriffe in der Schulterendoprothetik
}

\author{
Robert Hudek, Maik Zitzmann, Frank Gohlke
}

\section{Zusammenfassung}

Revisionseingriffe in der Schulterendoprothetik stellen höchste Ansprüche an das gesamte Team bez. Operatigrund der häufig komplexen Ausgangslage sind eine ausreichende Analyse der präoperativen Situation und die Auswahl geeigneter Wechselkomsamte Team einzubeziehen, da Rückzugsoptionen und ein schneller Verfahrenswechsel sichergestellt sein müssen. Die wesentlichen Wechselursachen sind mechanische Komplikationen durch Lockerung, Komponentenversagen oder Fraktur, Infektionen oder die Insuffizienz der Rotatorenmanschette nach der Implantation anatomischer Prothesen. Jedes dieser Versagensmuster verlangt eine spezifische Behandlung und Berücksichtigung unterschiedlicher Faktoren zur Erzielung einer funktionellen Verbesserung. Zur Durchführung muss ein spezielles Instrumentarium zur Verfügung stehen, mit dessen Anwendung das gesamte Team vertraut sein sollte. Infektionen sind durch den teilweisen oder kompletten Wechsel der Komponenten zu beherrschen. Hierbei kann eine antibiotikabeladene Interimsprothese je nach Ausmaß der Infektion notwendig werden. onsplanung und Durchführung. Aufponenten essenziell. Dabei ist das ge-

\section{Revision Operations on Total Shoulder Arthroplasties}

The surgical planning and performance of revision operations on total shoulder arthroplasties place the highest demands on the entire team. On account of the often complex starting conditions, an adequate analysis of the preoperative situation and the choice of suitable revision components are mandatory. The entire team must be involved as options for reversal and/or a rapid change in the procedures must be available. The main reasons for a procedural change are mechanical complications due to loosening, component failures or fractures, infections or insufficiency of the rotator cuff after implantation of an anatomic prosthesis. Each of these reasons for failure requires a specific management and consideration of widely varying factors in order to achieve an improvement in function. Special instruments must be available and the entire team should be familiar with their use. Infections should be managed by partial or complete exchange of the components. In such cases an interim prosthesis charged with antibiotics may be necessary depending on the extent of the infection.

stellt hohe Anforderungen an das gesamte Operationsteam, und die Erfahrungen im Umgang mit Revisionseingriffen sind in der Literatur entsprechend limitiert $[10,11]$. prothetik zählen zu den komplexesten Eingriffen und sind selbst für sehr erfahrene Schulterchirurgen eine Herausforderung $[9,11,18]$. Deren Durchführung

OP-JOURNAL 2013; 29: 98-103

(c) Georg Thieme Verlag KG Stuttgart · New York DOI http://dx.doi.org/10.1055/s-0032-1328264
Die Ursachen, die zu einem Revisionseingriff führen, lassen sich der Häufigkeit nach in 3 hauptsächliche Kategorien einteilen: 1. fehlgeschlagene anatomische (Fraktur-)Prothesen (Insuffizienz der Rotatorenmanschette, Einsteifung),
2. Infektionen (Früh- oder Spätinfekte), 3. mechanische Komplikationen (Lockerung, Instabilität, Fehlpositionierung, Materialbruch, Fraktur, Komponentenversagen).

Der präoperativen Planung fällt dabei eine besondere Rolle zu (Tab. 1). Sonderimplantate müssen teilweise anhand patientenspezifischer Merkmale und Bilddaten (CT, 3-D-Rekonstruktionen, Röntgenprojektionen mit Vergleich zur Gegenseite) bestellt werden. Bei einem Wechsel der Schaftkomponenten sind zudem spezielle Instrumentarien notwendig (Abb. 1) [11].

Für jeden Revisionseingriff gilt es, je nach zugrunde liegender Pathologie besondere Faktoren zu berücksichtigen, damit die Funktionsverbesserung durch den Wechsel gelingt. Die 3 Hauptkategorien sollen nachfolgend hinsichtlich ihrer Diagnostik und Planung sowie der handwerklichen Durchführung behandelt werden.

\section{Hauptteil}

\section{Mechanische Komplikationen}

In der Schulterendoprothetik kommen im Wesentlichen 3 Implantattypen zum Einsatz: 1. die anatomische Prothese, 2. der Oberflächenersatz, 3. die inverse Prothese. Die klassische anatomische Prothese besteht einerseits aus einem Schaft, der entweder zementiert oder unzementiert im Humerus verankert ist. Andererseits aus einem Glenoidersatz (Pfannenersatz), der aus hochvernetztem, abriebfestem Polyethylen (PE) hergestellt ist und in der Regel in die Pfanne zementiert wird [14]. Schaftlose Prothesen kommen mit einer zementfreien Verankerung in der proximalen Humerusmetaphyse aus [3]. Als Metaphyse bezeichnet man den dichten und aus schwammartiger Knochensubstanz (Spongiosa) aufgebauten Knochenabschnitt zwischen Diaphyse (Knochen- 


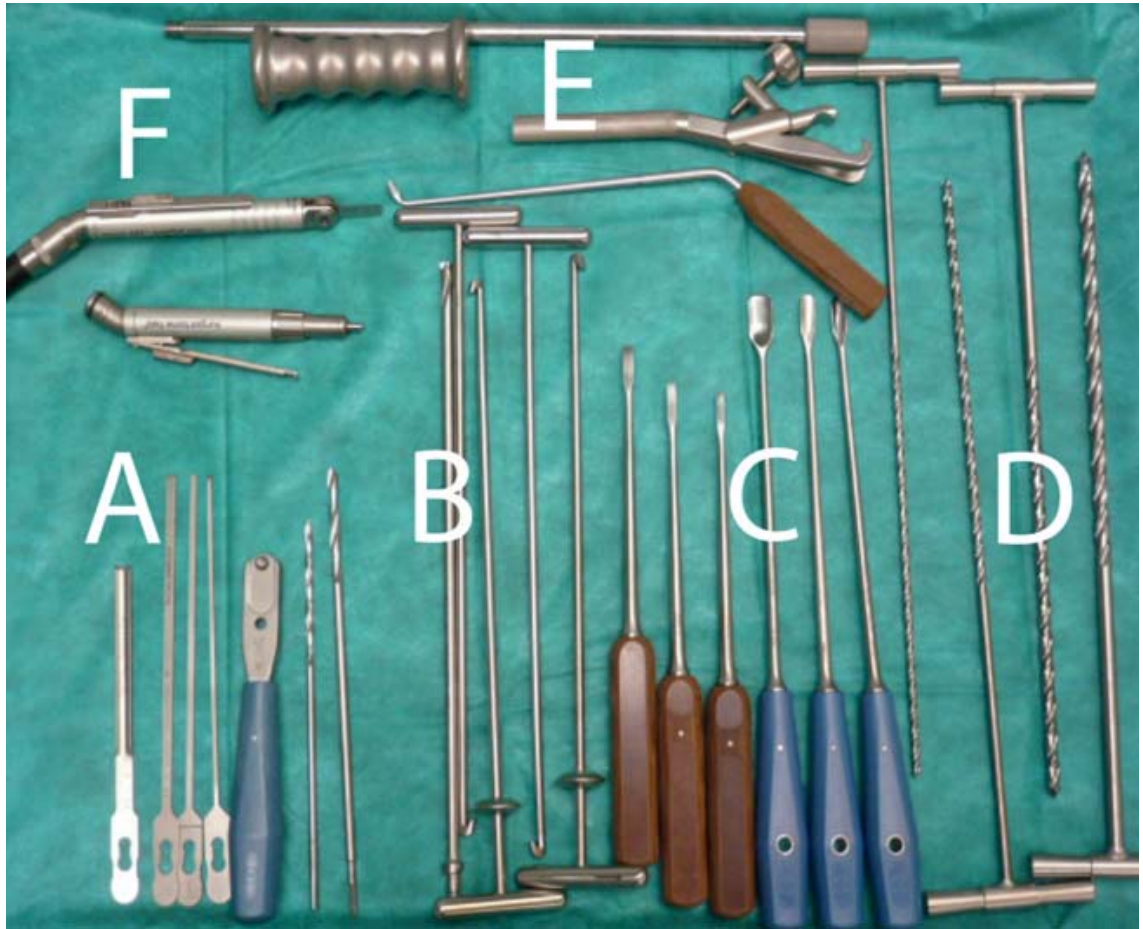

Abb. 1 a bis $\mathbf{f}$ Spezialinstrumentarien zum Prothesenwechsel und zur schonenden Entfernung der verbleibenden Knochenzementreste: a feine Meißel, Klingenmeißel, Bohrer; b Räumhaken; c Zementmeißel; d Handbohrer; e Ausschlaginstrumentarium; f Druckluftsäge/-fräse.

schaft) und Epiphyse (Kopf). Die häufigste Indikation für den Wechsel einer anatomischen auf eine inverse Prothese stellt eine fehlgeschlagene Frakturendoprothese mit schmerzhafter Pseudoparalyse dar [7].

Die Prothesentypen können gemäß ihrer biomechanischen Eigenschaften und Implantationstechniken unterschiedlich versagen. Nicht zuletzt können auch herstellerspezifische Versagensmuster auftreten, die bei neueren Implantaten ohne Langzeiterfahrung noch gar nicht bekannt sein müssen.

Bei anatomischen Prothesen wird die mittlere Komplikationsrate zwischen $10 \%$ und $16 \%$ angegeben $[5,8]$. Das kumulative Risiko wird in der Literatur bei $12 \%$ nach 5 Jahren angegeben [8]. Anteilig an der Gesamtzahl der Komplikationen wird die Lockerung der Glenoidkomponente (32\%) als der häufigste Versagensgrund angeführt, gefolgt von Instabilität (30\%) und periprothetischer Fraktur (11\%) [5]. Der Schaft bleibt in der Regel über einen sehr langen Zeitraum fest verankert und verursacht selten $(6,5 \%)$ mechanische Komplikationen [5]. Bei der Glenoidlockerung berichten die Patienten über zunehmende, belastungsabhängige Schmerzen nach einem teils jahrzehntelangen beschwerdefreien
Intervall [23]. Eine konventionelle Röntgenkontrolle oder Computertomografie (CT) zeigt dann Osteolysezonen um die Glenoidkomponente. Falls Unsicherheit hinsichtlich einer Lockerung besteht, so lässt sich mittels einer Schulterarthroskopie leicht die mechanische Stabilität des Glenoids beurteilen. Gleichzeitig können mikrobiologische und histologische Proben entnommen werden, um nach einer Keimbesiedelung der Prothese zu fahnden. Wenn eine Glenoidlockerung bestätigt wurde, so besteht bei Infektfreiheit prinzipiell die Indikation zu deren einzeitigem Wechsel. Als Revisionsglenoide werden entweder PE-Modelle oder solche mit einer Basis aus Metall („metal-backed“) verwendet, die im knöchernen Anteil verschraubt werden können [6]. Der Zugang zum gelockerten Glenoid kann jedoch erschwert sein, da die Kopfkomponente (Kalotte) nicht immer einwandfrei zu entfernen ist. Es sollten daher verschiedene Meißel (schmale Klingenmeißel, Zemententfernungsmeißel) vorhanden sein, da knöcherne Anteile der Metaphyse und Zementverbindungen vorsichtig entfernt werden müssen, um die Integrität des Schaftes (sofern er belassen werden kann) nicht zu gefährden. Besondere Bedeutung kommt der Füllung des knöchernen Defekts zu, der durch Osteolysen und Entfernung von PE und Zement am knö- chernen Glenoid entstanden ist. Der knöcherne Aufbau kann aus einem kortikospongiösen Span (Beckenkamm) erfolgen. Vor der Entnahme kann die benötigte Größe anhand von CT-Bilddaten grob kalkuliert werden. Falls ein Revisionsglenoid mit metallischer Basis zur Schraubfixation benutzt wird, kann der Span darunter unter Schraubenkompression eingefügt werden. Falls eine stabile Verankerung nicht möglich ist, muss zweizeitig vorgegangen und die ossäre Integration des kortikospongiösen Spans im Glenoid abgewartet werden. In einer Folgeoperation kann dann bei gesicherter knöcherner Konsolidierung ein neues Glenoid zementiert werden. Diese Methodik ist allerdings noch nicht hinreichend etabliert, und es existieren nur wenige Fallberichte $[6,21]$. Dennoch gilt es, bei komplexen Wechseln auch für diese Option gerüstet $\mathrm{zu}$ sein. Es sollten daher alle notwendigen Instrumente und Schrauben zur Verfügung stehen. Falls auf eine inverse Prothese gewechselt werden soll, so lässt sich der knöcherne Block direkt zwischen die Glenosphäre der inversen Prothese einsetzen, da diese im Gegensatz zu einem herkömmlichen PE-Glenoid winkelstabil unter Kompression verschraubt werden kann [15].

Hemiprothesen (Teilprothese) ohne Glenoidkomponente kommen als Schaftprothese, Kalottenersatz oder Oberflächenersatz vor. Meist kommen diese Modelle bei Folgezuständen nach Unfällen zum Einsatz. Schmerzhafte Bewegungseinschränkungen aufgrund posttraumatischer Nekrosen und frühzeitiger Arthrose am Humeruskopf sind die hauptsächlichen Indikationen [19]. Nach Implantation einer Hemiprothese kann es jedoch zur zunehmenden Erosion am Glenoid kommen, die eine 2. Operation zur Implantation einer PE-Glenoidkomponente zu einem späteren Zeitpunkt notwendig macht [13]. Falls ein Oberflächenersatz verwendet wurde, ist bei einer Wechsel-OP v.a. auf Osteolysen (Knochenauflösung) unter dem Implantat in der Humerusmetaphyse zu achten. Diese entstehen durch die veränderte Belastungseinleitung, die durch einen Oberflächenersatz hervorgerufen wird. Am Rand ist sie höher, dort bleibt der Knochen hart, im Zentrum ist sie geringer, dort löst er sich auf. Da bei ausgedehnten Osteolysen keine metaphysäre Verankerung mehr möglich ist, muss zum Wechsel eine zementierte Schaftprothese gewählt werden. Daher sollte bei der geplanten Implantation einer 
Tab. 1 Die Tabelle zeigt eine Übersicht zur Art des Wechseleingriffs in der linken Spalte. Die jeweiligen planungsrelevanten Punkte sind unter „Planung“ in der mittleren Spalte aufgeführt. Rechts stehen unter „Ausführung“ technische Aspekte, die den reibungslosen Ablauf im OP unterstützen sollen.

\begin{tabular}{|c|c|c|}
\hline Wechseleingriff & Planung & Ausführung \\
\hline Glenoidwechsel & $\begin{array}{l}\text { - Ausmessen Defektgröße \& } \\
\text { Glenoid (CT) } \\
\text { - Bestellung Revisionsglenoid } \\
\text { („metal-backed“) + Instru- } \\
\text { mentarium } \\
\text { - Beckenkammentnahme (Lage- } \\
\text { rung) }\end{array}$ & $\begin{array}{l}\text { - feine Meißel zur Entfernung von } \\
\text { Zement/Lösung der Kopfkalotte } \\
\text { - mehrere „Fukuda-Hebel“ (Expo- } \\
\text { sition Glenoid) } \\
\text { - mikrobiologische (Thio-Glykolat- } \\
\text { Lösung) und histologische Pro- } \\
\text { ben }\end{array}$ \\
\hline Hemiprothese & $\begin{array}{l}\text { - Wechsel auf anatomische } \\
\text { Schaftprothese oder inverse } \\
\text { Prothese (Rotatorenmanschet- } \\
\text { te intakt?) } \\
\text { - eventuell Konversion auf inver- } \\
\text { se Prothese (Hersteller) }\end{array}$ & $\begin{array}{l}\text { - Oberflächenersatz - oft mit Os- } \\
\text { teolysen der Metaphyse ver- } \\
\text { gesellschaftet } \\
\text { - schaftlose Prothesen - mehrere } \\
\text { Sägeblätter vorhalten, wenn } \\
\text { knöchern zu fest verankert } \\
\text { - Achtung bei der Glenoidexposi- } \\
\text { tion - Hebel können Metaphyse } \\
\text { brechen }\end{array}$ \\
\hline Schaftprothese & $\begin{array}{l}\text { - Ausmessen Schaftlänge (bis } \\
\text { Ende des Zementköchers, } \\
\text { Abb. 5) } \\
\text { - Länge Revisionsschaft bestim- } \\
\text { men - sollte mindestens um } \\
5 \text { cm länger als die Ausdeh- } \\
\text { nung des Zementköchers nach } \\
\text { unten sein (Hersteller mit lan- } \\
\text { gen Schäften bis } 210 \text { mm aus- } \\
\text { suchen) }\end{array}$ & $\begin{array}{l}\text { - mehrere Sägeblätter vorhalten, } \\
\text { da Schaft der Länge nach eröff- } \\
\text { net werden muss } \\
\text { - Kabelcerclagen vorhalten zur Re- } \\
\text { adaptation/Fixierung Tubercula } \\
\text { - } 2 \text { Blutkonserven parat halten } \\
\text { - Autotransfusion verwenden } \\
\text { - alle Größen von Meißeln und } \\
\text { Hochfrequenzfräse zur Zement- } \\
\text { entfernung parat halten } \\
\text { - mikrobiologische und histologi- } \\
\text { sche Proben entnehmen }\end{array}$ \\
\hline inverse Prothese & $\begin{array}{l}\text { - Ursachenfahndung (Ausrisse, } \\
\text { Frakturen, Schulterdach!) } \\
\text { - Deltamuskel \& N. axillaris (in- } \\
\text { takt?) } \\
\text { - Verankerung für Schrauben } \\
\text { suchen } \\
\text { - Glenoidausbrüche: Revision } \\
\text { unter Einsatz von Becken- } \\
\text { kammspan } \\
\text { - Zementspacer einplanen } \\
\text { - Basisplatte mit extralangem } \\
\text { Zapfen (20-30 mm) bereit- } \\
\text { stellen }\end{array}$ & $\begin{array}{l}\text { - Schrauben, Platten und Fraktur- } \\
\text { siebe bereit halten } \\
\text { - Beckenkammentnahme vor- } \\
\text { bereiten (Lagerung) } \\
\text { - mikrobiologische und histologi- } \\
\text { sche Proben entnehmen } \\
\text { - Material für Zementspacer parat } \\
\text { halten, wenn zweizeitiges Vor- } \\
\text { gehen }\end{array}$ \\
\hline
\end{tabular}

Glenoidkomponente bei Patienten mit Oberflächenersatz auch an die Möglichkeit zum Wechsel auf eine Schaftprothese gedacht werden.

Die Versagensmechanismen der inversen Prothese unterscheiden sich durch ihre biomechanischen Eigenschaften von denen der anatomischen Modelle [1,2]. Bei der inversen Prothese versagt v.a. die Basisplatte, oder es kann zum Anschlagen der metallischen Prothesenmetaphyse an die Skapula („scapular notching“) kommen, zum Komponentenbruch sowie zu Stressfrakturen von Akromion (Schulterdach) und Spina scapulae [1]. Dies trifft insbesondere auf ältere Patien- ten zu, die aufgrund von rheumatischen Erkrankungen und Osteoporose eine geringe Knochendichte aufweisen.

Bei unzureichender Implantationstechnik kann es zudem zu knöchernen Ausrissen und Frakturen des Skapulahalses oder zu Schraubenbrüchen im Bereich der Basisplatte kommen. Falls keine stabile Verankerung mehr möglich ist: Einbringen autogenen Knochens und artikulierender Spacer. Brüche von Akromion und Spina: Osteosynthese des Schulterdachs mittels winkelstabiler Platte oder Zuggurtung.

Wenn gar die Glenosphäre herausgebrochen ist, muss zunächst die knöcherne
Heilung des Skapulahalses herbeigeführt werden. Dies gelingt in der Regel nur durch eine Implantatentfernung und den knöchernen Aufbau des Glenoids oder Osteosynthese des Skapulahalses. Nur wenn ein ausreichender knöcherner Grundstock vorhanden ist, kann die erneute Implantation der Glenosphäre erfolgen.

\section{Infektionen}

Es werden in der Endoprothetik grundsätzlich nach ihrer Entstehung hämotogene, lokal fortgeleitete oder exogene Infekte (z. B. nach Injektionen) unterschieden. Von einer Frühinfektion spricht man, wenn sich im Zeitraum bis 3 Monate nach dem Ersteingriff eine Infektion bestätigt. Häufig erfolgt die Bestätigung durch einen positiven Keimnachweis im Rahmen einer arthroskopischen Probenentnahme. In diesen Fällen reicht häufig ein partieller Wechsel der unzementierten Komponenten (Kalotte, PEInlay, Glenosphäre) aus. Bei Frühinfekten haben Bakterien kaum Gelegenheit, sich tief im effektiven Gelenkraum zu verteilen und erreichen selten zementierte Schaftanteile [4].

Während des Komponentenwechsels müssen mehrere mikrobiologische Proben entnommen werden. Die Transportröhrchen sollten Thio-Glykolat-Lösung beinhalten, da schwer anzüchtbare anaerobe Keime wie z. B. Propionibacterium acnes sonst nicht nachweisbar sind [17].

Die Thio-Glykolat-Probenröhrchen werden vor der Entnahme gekühlt gelagert und sollten nach Beimpfung unmittelbar ins mikrobiologische Labor transportiert werden. Es sollten zudem histologische Proben der umliegenden Weichteile entnommen werden. Erst nach abgeschlossener Entnahme kann eine kalkulierte Verabreichung eines Breitspektrumantibiotikums erfolgen. Es ist darauf zu achten, dass auch anaerobe Keime wie Propionibacterium acnes durch das Wirkspektrum abgedeckt sind (z.B. Amoxicillin + Clavulansäure/Sulbactam). Bei Spätinfekten ist meist von einer hämatogenen Streuung auszugehen. Sie treten erst nach über 2 Jahren auf. Zusätzlich liegen häufig begünstigende Faktoren wie Immundefekte, ein Diabetes mellitus oder eine immunsuppressive Therapie (z.B. Kortison) bei rheumatischen Erkrankungen vor. Erreger eines Keimherds im Nasen-/Rachenraum, an oberflächlichen Hautdefekten 


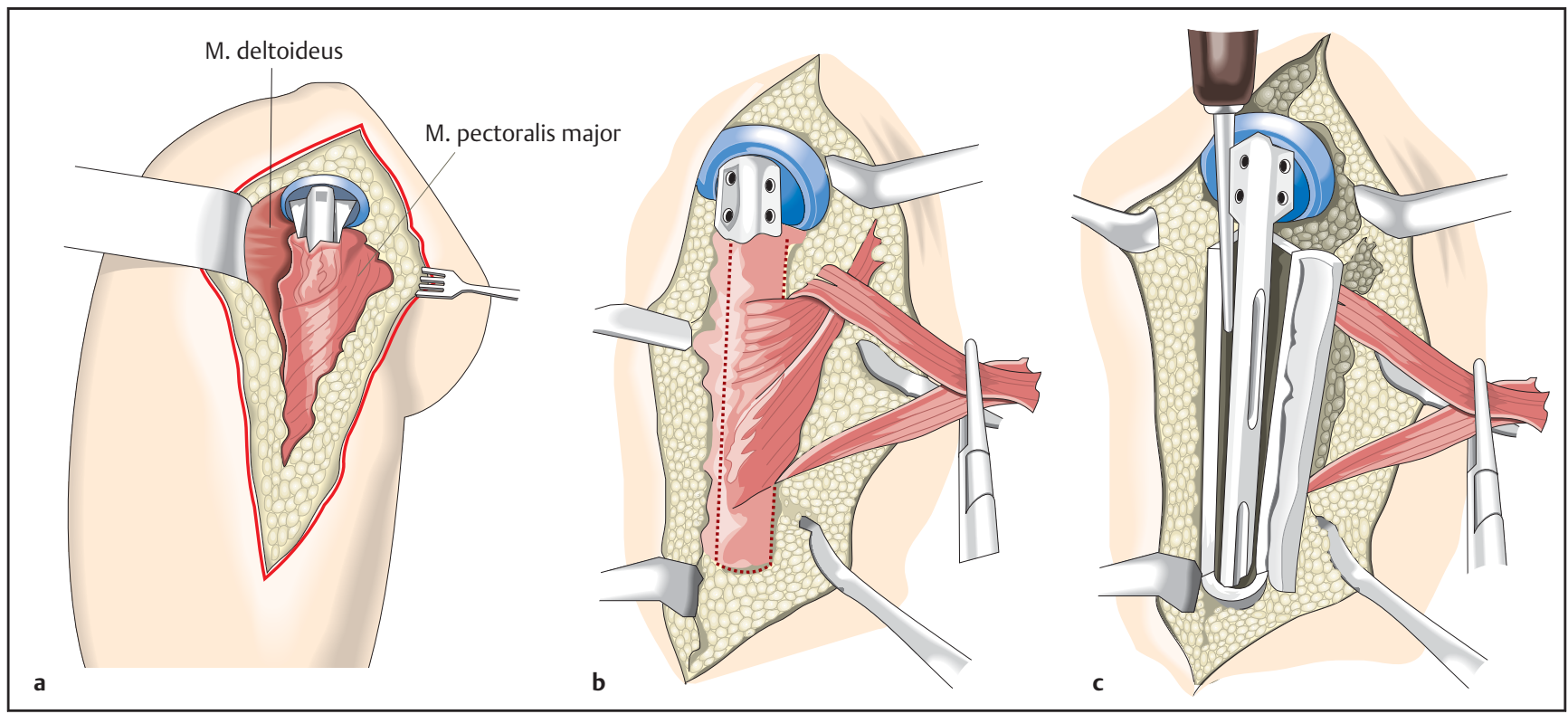

Abb. 2 Vorgehen beim Schaftwechsel: Es wird im deltoideopektoralen Intervall der Schaft dargestellt. Unter Erhalt des Ansatzes des M. pectoralis major am vorderen Humerusschaft wird mit einer oszillierenden Säge der Schaft entlang der roten Linie aufgesägt. Mit verschiedenen Meißeln kann der Schaft samt Zementköcher entfernt werden.

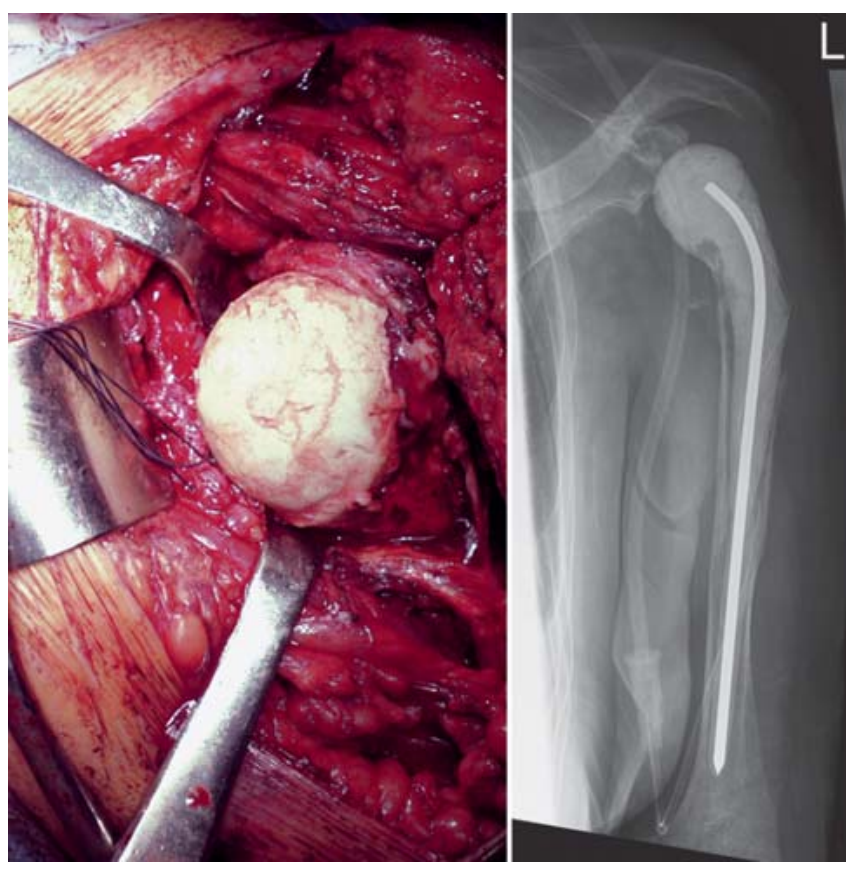

oder an Verdauungsorganen und Herzklappen können sich bei entsprechend schlechter Abwehrlage an der Prothese ansiedeln. Manche Keime bilden dort einen sog. „Biofilm“. Das ist ein „strukturierter Zusammenschluss bakterieller Zellen, die sich eingeschlossen in einer selbstproduzierten Matrix und adhärent zu inerten oder lebenden Oberflächen befinden“ [20]. Darunter können sich die Erreger sehr effektiv vor der Wirkung von Antibiotika verbergen [20]. Bei mehrjähriger Tragezeit einer Prothe-

Abb. 3 Platzhalter aus Knochenzement (PMMA) versetzt mit Gentamycin- und Vancomycinantibiotikum. Der Humeruskopf wird mit einer Schablone handgeformt nachempfunden, um möglichst anatomische Verhältnisse herzustellen. Dadurch soll auch der Abrieb am Glenoid verringert werden. Daneben das Röntgenbild; als zentrale Stütze im Platzhalter wirkt ein vorgebogener Gewindestab zu Verbesserung der Stabilität.

Robert Hudek et al.: Revisionseingriffe in der Schulterendoprothetik enkraum [22]. Abriebpartikel aus Polymehrerer Jahre tief zwischen metallische Teile und Zementköcher vordringen. Bei einem Spätinfekt ist meist der dann gelingen, das vorbestehende Implantat samt Zementköcher zu entfernen, ohne die knöcherne Substanz des Humerusschafts oder dessen Durchblu- tung zu erodieren. Der Schaft wird der Länge nach unter Erhalt des Ansatzes des M. pectoralis major aufgesägt (Abb. 2 und 4) [11]. Der Erhalt dieses Ansatzes im vorderen Anteil des Humerusschafts sichert die Durchblutung des entstandenen knöchernen Fensters. Nach der gründlichen Spülung sämtlicher Gelenkräume per Jet-Lavage mit desinfizierender Lösung (z.B. Lavanid ${ }^{\circledR}$ ) und anschließender Spülung mit Ringer-Lösung erfolgt die Implantation eines Platzhalters aus Knochenzement (Abb. 3). Dieser wird in unserer Abteilung per Hand geformt und mit Gentamycin-/Vancomycinantibiotika angereichert. Diese entfalten ihre Wirkung unmittelbar in der Markraumhöhle und augmentieren die nachfolgende mehrwöchige Antibiotikatherapie (ca. 8 Wochen) [20]. Erst wenn klinisch und laborchemisch die Infektfreiheit angenommen werden kann, kann der Wechsel auf ein Revisionsmodell erfolgen. In der Regel wird dann auf eine inverse Prothese zurückgegriffen, da nach ausgedehnten Infekten die Rotatorenmanschette häufig zerstört ist.

\section{Fehlgeschlagene anatomische Prothesen}

Die anatomische Totalprothese ist im Gegensatz zur inversen Prothese dem menschlichen Schultergelenk nachempfunden. Sie besteht aus Schaft mit Kopfkalotte und Glenoidkomponente. Eine intakte Rotatorenmanschette, eine Gruppe von 4 Muskeln und Sehnen, ist 


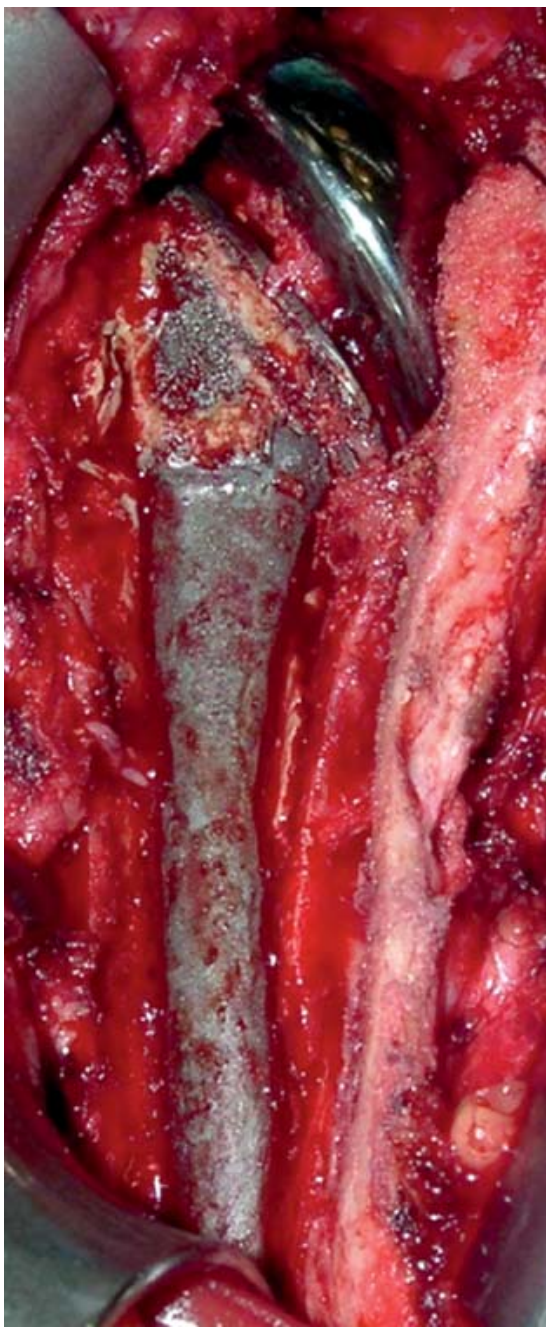

Abb. 4 Intraoperative Aufnahme einer Schaftrevision mit zementiertem Schaft einer anatomischen Prothese. Das Knochenfenster ist bereits aufgesägt, der Schaft in ganzer Länge dargestellt. Als nächster Schritt erfolgt die schonende Entfernung des Schaftes unter Einsatz von Meißeln, Säge und Fräse.

zur Funktion dieser Konstruktion unabdingbar [14]. Die Manschette zentriert den Humeruskopf in der Pfanne und leitet die Bewegungen der Schulter ein. Wenn ein oder mehrere Anteile dieser Sehnen rupturieren oder degenerieren, kann die Schulterfunktion nicht mehr hinreichend aufrechterhalten werden. Anatomische Prothesen sollten daher nur bei Patienten mit intakter Rotatorenmanschette implantiert werden [14]. Nach einer Prothesenimplantation kann es degenerativ oder traumatisch $\mathrm{zu}$ einem Verlust der Sehnensubstanz kommen [5]. Insgesamt ist die anterosuperiore Dezentrierung die häufigste Manifestation einer postoperativen Instabilität [5] und in der Regel auf Defekte des vorderen Anteils der Rotatorenman-

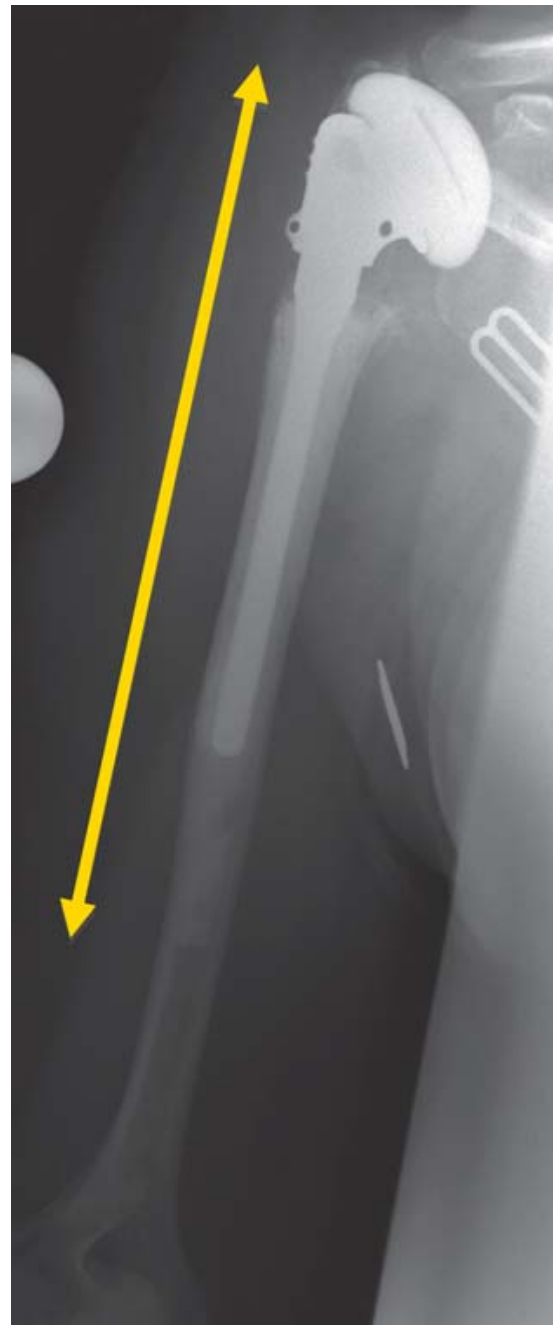

Abb. 5 Ausmessen der einliegenden Prothese. Es muss ein Röntgenbild vorliegen, das den gesamten Humerus zeigt, da Zementanteile bis weit nach distal vordringen können. Ein zementierter Revisionsschaft sollte um 5 cm länger sein als das distale Ende des Zementköchers.

schette, den M. subscapularis, zurückzuführen. Dieser versperrt den Zugang zum Gelenk und muss bei der Implantation zunächst abgetrennt und nach Implantation wieder angenäht werden.

Falls eine traumatische Ursache für einen Sehnenriss vorliegt, kann eine zeitnahe Rekonstruktion der Sehne versucht werden. Das Ergebnis ist allerdings ungewiss, eine zügige Revision nach dem Trauma führt tendenziell $\mathrm{zu}$ besseren Ergebnissen $[12,16]$. Falls sich der Sehnenverlust schleichend vollzogen hat und eine Rekonstruktion nicht mehr möglich ist, so bleibt nur der Wechsel auf ein inverses Modell. Hier kann aufgrund der invertierten Konstruktion und besonderer biomechanischer Eigenschaften auf die
Funktion der Rotatorenmanschette verzichtet werden. Falls das implantierte anatomische Modell herstellerseitig auf ein inverses Modell konvertiert werden kann, so lässt sich der Prothesenschaft im Humerus belassen. Eine Umwandlung ist daher wesentlich einfacher durchzuführen, da die metaphysäre Komponente umgesteckt werden kann. Wenn keine Konversion möglich ist, so bleibt nur der Schaftwechsel unter Aufsägen eines knöchernen Fensters (Abb. 2). Für das OP-Team ist es von entscheidender Bedeutung, dass die Wechselkomponenten und Instrumentarien sicher bedient werden können. Falls die implantierte Prothese nicht zu dem hauseigenen gängigen Sortiment zählen sollte, empfiehlt es sich, einen versierten Mitarbeiter des Herstellers zur Operation einzubestellen, um allfällige Bedienungsfragen prompt beantworten zu können.

\section{Schlussfolgerung}

Revisionseingriffe in der Schulterendoprothetik stellen hohe Ansprüche an das gesamte OP-Team. Die präoperative Planung und die Möglichkeit zum raschen Verfahrenswechsel beim Ausschöpfen von Rückzugsoptionen sind wichtige Elemente. Die Versagensgründe unterscheiden sich je nach Prothesentyp und machen einen partiellen oder gesamten Wechsel notwendig. Fehlgeschlagene anatomische Implantate lassen sich bei Defekten der Rotatorenmanschette auf eine inverse Prothese wechseln, Protheseninfekte lassen sich durch einen partiellen oder totalen Komponentenwechsel beherrschen, die Behandlungsdauer kann jedoch mehrere Monate in Anspruch nehmen. Je nach Ausmaß muss temporär eine antibiotikabeladene Interimsprothese eingesetzt werden.

\section{Literatur}

${ }^{1}$ Affonso J, Nicholson GP, Frankle MA, Walch G, Gerber C, Garzon-Muvdi J, McFarland EG. Complications of the reverse prosthesis: prevention and treatment. Instr Course Lect 2012; 61: 157-168

2 Alta TD, Veeger HE, Janssen TW, Willems WJ. Are shoulders with a reverse shoulder prosthesis strong enough? A pilot study. Clin Orthop Relat Res 2012; 470: 2185-2192

3 Berth A, Pap G. Stemless shoulder prosthesis versus conventional anatomic shoulder prosthesis in patients with osteoarthritis: a comparison of the functional outcome after a minimum of two years follow-up. J Orthop Traumatol 2013: 14: 31-37

${ }^{4}$ Betsch BY, Eggli S, Siebenrock KA, Tauber MG, Muhlemann K. Treatment of joint prosthesis infection in accordance with current recommendations improves outcome. Clin Infect Dis 2008; 46: 1221-1226 
${ }^{5}$ Bohsali KI, Wirth MA, Rockwood jr. CA. Complications of total shoulder arthroplasty. J Bone Joint Surg [Am] 2006; 88: 2279-2292

6 Bonnevialle N, Melis B, Neyton L, Favard L Mole D, Walch G, Boileau P. Aseptic glenoid loosening or failure in total shoulder arthroplasty: revision with glenoid reimplantation. J Shoulder Elbow Surg 2012 Oct 30 [Epub ahead]

7 Carroll RM, Izquierdo R, Vazquez M, Blaine TA Levine WN, Bigliani LU. Conversion of painful hemiarthroplasty to total shoulder arthroplasty: long-term results. J Shoulder Elbow Surg 2004; 13: 599-603

${ }^{8}$ Chin PY, Sperling JW, Cofield RH, Schleck C. Complications of total shoulder arthroplasty: are they fewer or different? J Shoulder Elbow Surg 2006; 15: 19-22

${ }^{9}$ Cofield RH, Edgerton BC. Total shoulder arthroplasty: complications and revision surgery. Instr Course Lect 1990; 39: 449-462

10 De Wilde L, Mombert M, Van Petegem P, Verdonk $R$. Revision of shoulder replacement with a reversed shoulder prosthesis (Delta III): report of five cases. Acta Orthop Belg 2001; 67: 348-353

11 Gohlke F, Rolf $O$. [Revision of failed fracture hemiarthroplasties to reverse total shoulder prosthesis through the transhumeral approach: method incorporating a pectoralismajor-pedicled bone window]. Oper Orthop Traumatol 2007; 19: 185-208

12 Hattrup SJ, Cofield RH, Cha SS. Rotator cuff repair after shoulder replacement. J Shoulder Elbow Surg 2006; 15: 78-83
${ }^{13}$ Levine WN, Fischer CR, Nguyen D, Flatow EL, Ahmad CS, Bigliani LU. Long-term follow-up of shoulder hemiarthroplasty for glenohumeral osteoarthritis. J Bone Joint Surg [Am] 2012; 94: e164

${ }^{14}$ Liem D, Marquardt B, Witt KA, Steinbeck J. [Shoulder arthroplasty: biomechanics and design]. Orthopade 2007; 36: 1027-1036

${ }^{15}$ Melis B, Bonnevialle N, Neyton L, Levigne C, Favard L, Walch G, Boileau P. Glenoid loosening and failure in anatomical total shoulder arthroplasty: is revision with a reverse shoulder arthroplasty a reliable option? J Shoulder Elbow Surg 2012; 21: 342-349

${ }^{16}$ Miller BS, Joseph TA, Noonan TJ, Horan MP, Hawkins RJ. Rupture of the subscapularis tendon after shoulder arthroplasty: diagnosis, treatment, and outcome. J Shoulder Elbow Surg 2005; 14: 492-496

${ }^{17}$ Nielsen PA. Role of reduced sulfur compounds in nutrition of Propionibacterium acnes. J Clin Microbiol 1983; 17: 276-279

18 Petersen SA, Hawkins RJ. Revision of failed total shoulder arthroplasty. Orthop Clin North Am 1998; 29: 519-533

${ }^{19}$ Rasmussen JV, Jakobsen J, Brorson S, Olsen BS. The Danish Shoulder Arthroplasty Registry: clinical outcome and short-term survival of 2,137 primary shoulder replacements. Acta Orthop 2012; 83: 171-173

${ }^{20}$ Rolf O, Stehle J, Gohlke F. [Treatment of septic arthritis of the shoulder and periprosthetic shoulder infections. Special problems in rheumatoid arthritis]. Orthopade 2007; 36: 700-707
${ }^{21}$ Scalise JJ, Iannotti JP. Bone grafting severe glenoid defects in revision shoulder arthroplasty. Clin Orthop Relat Res 2008; 466: 139-145

22 Schmalzried TP, Jasty M, Harris WH. Periprosthetic bone loss in total hip arthroplasty. Polyethylene wear debris and the concept of the effective joint space. J Bone Joint Surg [Am] 1992; 74: 849-863

23 Strauss EJ, Roche C, Flurin PH, Wright T, Zuckerman JD. The glenoid in shoulder arthroplasty. J Shoulder Elbow Surg 2009; 18: 819-833

\section{Dr. med. Robert Hudek \\ Assistenzarzt \\ Maik Zitzmann \\ OP-Leitung \\ Prof. Dr. med. Frank Gohlke \\ Chefarzt}

Abteilung für Schulterchirurgie Rhön Klinikum AG

Salzburger Leite 1

97616 Bad Neustadt/Saale

robert@hudek.de 